\title{
Linear Energy Transfer Dependence Correction of Spread-Out Bragg Peak Measured by EBT3 Film for Dynamically Scanned Proton Beams
}

\author{
Moonhee Lee ${ }^{1(\infty)}$, Sunghwan Ahn ${ }^{2 \oplus}$, Wonjoong Cheon ${ }^{1, *(1)}$, Youngyih Han ${ }^{1,2 \oplus}$ \\ ${ }^{1}$ Samsung Advanced Institute for Health Sciences \& Technology (SAIHST), Sungkyunkwan University, ${ }^{2}$ Department of Radiation Oncology, \\ Samsung Medical Center (SMC), Seoul, Korea
}

Received 15 July 2020

Revised 17 October 2020

Accepted 2 November 2020

Corresponding author

Youngyih Han

(youngyih@skku.edu)

Tel: 82-2-3410-2604

Fax: 82-2-3410-2619

*Present address: Department of Radiation Oncology, National Cancer Center (NCC), Goyang 10408, Korea
Purpose: Gafchromic films for proton dosimetry are dependent on linear energy transfers (LETs), resulting in dose underestimation for high LETs. Despite efforts to resolve this problem for singleenergy beams, there remains a need to do so for multi-energy beams. Here, a bimolecular reaction model was applied to correct the under-response of spread-out Bragg peaks (SOBPs).

Methods: For depth-dose measurements, a Gafchromic EBT3 film was positioned in water perpendicular to the ground. The gantry was rotated at $15^{\circ}$ to avoid disturbances in the beam path. A set of films was exposed to a uniformly scanned 112-MeV pristine proton beam with six different dose intensities, ranging from 0.373 to $4.865 \mathrm{~Gy}$, at a 2-cm depth. Another set of films was irradiated with SOBPs with maximum energies of 110,150 , and $190 \mathrm{MeV}$ having modulation widths of $5.39,4.27$, and $5.34 \mathrm{~cm}$, respectively. The correction function was obtained using 150.8-MeV SOBP data. The LET of the SOBP was then analytically calculated. Finally, the model was validated for a uniform cubic dose distribution and compared with multilayered ionization chamber data.

Results: The dose error in the plateau region was within $4 \%$ when normalized with the maximum dose. The discrepancy of the range was $<1 \mathrm{~mm}$ for all measured energies. The highest errors occurred at $70 \mathrm{MeV}$ owing to the steep gradient with the narrowest Bragg peak.

Conclusions: With bimolecular model-based correction, an EBT3 film can be used to accurately verify the depth dose of scanned proton beams and could potentially be used to evaluate the depth-dose distribution for patient plans.

Keywords: Proton beam, Spread-out Bragg peak, Depth dose, Linear energy transfer, EBT3 film

\section{Introduction}

In proton therapy using a dynamically scanned beam, the desired depth-dose distribution is composed of stacked beam layers with unique energies, and each layer is dynamically scanned with a pencil beam that is magnetically deflected [1]. Despite having advantages over the use of passive scattered beams [2], the verification of depth-dose distribution using an ionization chamber, which is a point- by-point detector, is extremely inefficient and laborious. Devices with multiple ionization chambers, such as multilayer ionization chambers (MLICs), enable a shorter measurement time owing to their short setup time and rapid estimation of depth dose. Therefore, MLICs have been used in many facilities to obtain depth-dose data. However, MLICs are expensive devices and have a detector spacing of $2 \mathrm{~mm}$, which may be insufficient to provide the required spatial resolution for a low-energy proton beam, owing to the high- 
gradient distal depth dose. Moreover, MLICs only allow for measurements of fields smaller than $10 \times 10 \mathrm{~cm}$, rendering them unsuitable for measuring the depth dose of large field sizes. Therefore, a backup measurement system for depthdose profiles that complements the limitations of MLICs is required.

Gafchromic EBT3 film (Ashland, Covington, GA, USA) possesses a high spatial resolution down to $25 \mu \mathrm{m}$, which is particularly beneficial for the high-dose-gradient region of proton beams. By placing the film along the direction of the dynamically delivered beam, the depth-dose distribution can be determined with a single irradiation, rendering it an extremely time-efficient tool compared with point-bypoint dosimeters, which require a number of irradiations. However, the energy dependence of Gafchromic EBT3 film is still a critical issue in proton dosimetry, as proton beams undergo a significant change in energy and linear energy transfer (LET) along their paths, particularly in the Bragg peak region, where protons lose most of their energy and then stop, immediately after intensively depositing energy [3]. The energy dependence of the film leads to an underdose response in the Bragg peak region [4-6]. Therefore, determining an optimal method for correcting the underdose effect is essential to allow for the use of Gafchromic EBT films in the depth-dose measurement of proton beams.

The protocol for the calibration and dosimetry of EBT films provided by the manufacturer states that the calibration or characteristic curves are to be achieved by relating the film responses and the corresponding dose measurement results from a well-calibrated exposure, meaning it is necessary to apply a fitting function to the plot. However, a one-dimensional (1D) calibration function used to determine the absorbed dose based on film response cannot account for the implicit physics involved regarding the energy dependence of the film in the proton beam. Thus, conventional calibration methods for proton beams have resulted in underestimations of doses in the distal area.

Several researchers have attempted to resolve this underdosing issue [6-12]. In particular, numerical or mathematical correction methods that do not involve physical representation of the LET effect have been widely suggested. These methods complicate the correction of complex beam compositions, and therefore, previous studies have mainly focused on single-energy beams or a particular range of energies. Zhao and Das [7] reported polynomial fitting function, which is only based on a pristine Bragge peak, as a correction method for EBT3 films and evaluated the function by applying it to eight different energies ranging from 76 to $186 \mathrm{MeV}$. Park et al. [9] attempted to correct spreadout Bragg peaks (SOBPs) by separately correcting the data of each of the single-energy beams composing the SOBP beam. Subsequently, they combined these with a weighting function based on pristine Bragg peaks, thereby requiring a measurement for each single-energy beam with several sheets of films and the respective corrections [9]. Gambarini et al. [10] presented a method that uses the ratio of the percentage depth dose (PDD), measured using an ionization chamber, to the EBT3 film as a correction factor. They used the method on a multi-energy beam ranging from 62.73 to $81.56 \mathrm{MeV}$, which comprises the low-energy range in proton therapy [10]. Fiorini et al. [11,12] suggested a correction method for SOBP beams based on Monte Carlo simulations using a beam quality correction factor. The beam quality factor was successfully used to rectify underestimated doses for SOBPs. However, it was benchmarked using a $29-\mathrm{MeV}$ modulated beam that is only applicable to low-energy applications, such as eye treatments. Perles et al. [8] suggested a bimolecular model as a method for film response-to-dose calibrations using an LET correction-incorporated system, as the radiation-induced chemical reaction of radiochromic films occurred between two adjacent monomers within the same crystal lattice. This theoretically derived formalism is based on the law of mass action, which accounts for the reaction rate of materials. The bimolecular model is valid within a $5 \%$ accuracy for the depth dose of an $85.6-\mathrm{MeV}$ beam [8].

For the bimolecular model to be clinically applicable, further studies are required to extend the depth-dose measurements to multi-energy beams for a diverse range of energies. In this study, a method was thus developed for multi-energy beams with a larger energy range (i.e., 70-190 $\mathrm{MeV}$ ), with a focus on clinical applications. To achieve this, an SOBP was formed by a number of different energy layers. Overall, the aim of this study was to devise a correction method for the sum of the beam layers. 
Table 1. Specifications of irradiated proton beams

\begin{tabular}{|c|c|c|c|c|c|c|c|c|}
\hline Specification & \multicolumn{5}{|c|}{ Pristine Bragg peak } & \multicolumn{3}{|c|}{ SOBP } \\
\hline Snout Position (mm) & \multicolumn{5}{|c|}{400} & \multicolumn{3}{|c|}{400} \\
\hline Collimator $(\mathrm{cm} \times \mathrm{cm})$ & \multicolumn{4}{|c|}{$10 \times 10$} & & \multicolumn{3}{|c|}{$10 \times 10$} \\
\hline Energy/max. Energy of SOBP (MeV) & 70 & 92 & 112 & 150 & 190 & 110 & 150 & 190 \\
\hline Modulation width of SOBP $(\mathrm{cm})$ & & & - & & & 5.39 & 4.27 & 5.34 \\
\hline
\end{tabular}

SOBP, spread-out Bragg peaks.

\section{Materials and Methods}

\section{Theoretical concept of bimolecular model for film dosimetry}

The bimolecular model has been proposed as a correction method for the energy dependence of EBT2 films [8] and is generally presented as a formal derivation of the Schumann plate equation. The Schumann plate equation was originally derived for the detection of ultraviolet radiation using photographic plates [13] and is expressed as shown in Eq. (1). Subsequently, Candler [14,15] used the law of mass action to derive the Schumann equation by modeling a diatomic reaction. Regarding the chemical reaction to a radiation dose, at least two adjacent monomers must provoke a chemical response, or polymerization, which is modeled as a bimolecular chemical reaction. Perles et al. [8] modified the bimolecular model to account for the LET effect by making the reaction cross-section constant a function of the LET. In this model, the Schumann plate equation is used to represent the relationship between the dose and film response, with consideration of LET dependence.

$$
\frac{O D}{O D-O D_{\max }}=\left(\frac{D}{D_{1 / 2}}\right)^{p}
$$

It is noteworthy that the parameters in the bimolecular model are represented differently from those in the original Schumann plate equation. In Eq. (1), $O D$ is the optical density that characterizes the film response, and $p$ is the reaction order, which is an experimentally determined dimensionless parameter. $O D_{\max }$ is a constant proportional to the maximum value of $O D$ and is scanner dependent. Both $O D_{\max }$ and $p$ are empirically determined constant values. $D_{1 / 2}$ is a parameter determined by the cross section of a bimolecular reaction, and it indicates the LET effect. There- fore, $D_{1 / 2}$ can be presented as a function of LET or as a correction factor for dose underestimation.

\section{Proton beam system}

Film measurements were performed using dynamic linescanned beams comprising single and multiple energies to form an SOBP using the Sumitomo Proton Therapy System (Sumitomo Heavy Industries, Tokyo, Japan) at the Proton Therapy Center of the Samsung Medical Center (SMC, Seoul, Korea). To measure the pristine Bragg peaks, beams of energy ranging from 70 to $190 \mathrm{MeV}$, as well as a $112-\mathrm{MeV}$ beam with six different dose intensities ranging from 0.373 to $4.865 \mathrm{~Gy}$, were used. Furthermore, SOBPs were produced with three different maximum energies with modulation widths of 5.39, 4.27 , and $5.34 \mathrm{~cm}$. The field size of all irradiated beams was $10 \times 10 \mathrm{~cm}$. For continuous line-scanned beams, the dose intensity was determined by the scan speed $(\mathrm{mph})$ and dose rate (MU/s). Once the range and modulation width were determined, an SOBP was created by consecutively scanning each layer with different energies and dose weights of the SOBP. For beam irradiations, the scan speed was changed according to the dose weight, whereas the dose rate was fixed for all energies. The irradiated beams are listed in Table 1.

\section{Film measurement system}

Gafchromic EBT3 film (Ashland ISP Advanced Materials, Wayne, NJ, USA) was used to obtain the depth-dose distribution. All measurements were performed using film sheets from the same batch (Lot no. 05181501). To obtain the depth-dose profile, the film was positioned perpendicular to the ground and fixed by an in-house-designed film holder, as shown in Fig. 1. The in-house-designed phantom was composed of an acrylic framework and bolts. The films 

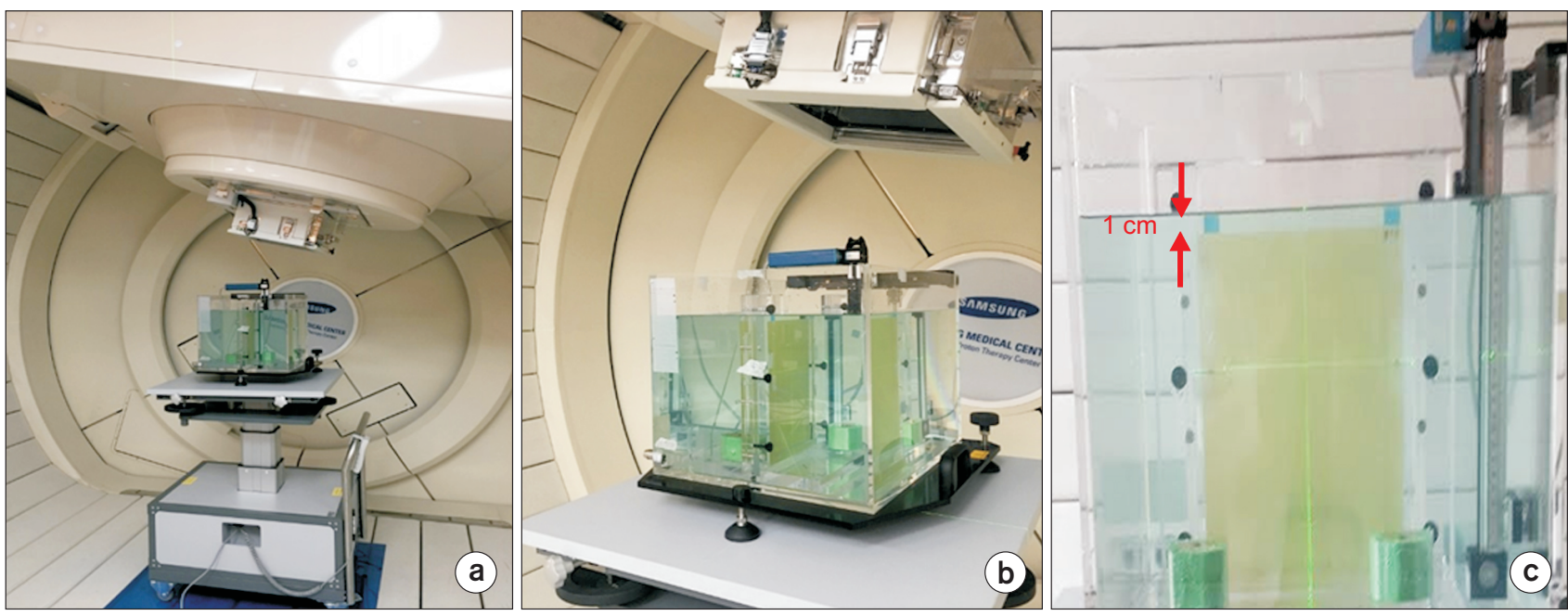

Fig. 1. (a) Experimental setup of the film fixed by an in-house-designed film holder in a water tank, (b) Gantry tilted as 3 degree, (c) $1 \mathrm{~cm}$ of the gap distance between water surface and the edge of the film.

could be inserted easily into the holder, even in water, and the bolts were tightened to ensure that the EBT3 film was fixed firmly. However, if the bolts were fixed too tightly, it was difficult to insert the film.

Because the film was set parallel to the beam axis in water, perturbations occurred in the proton beam range. To achieve an optimum range accuracy, it is essential to avoid perturbations. First, the film was completely immersed in water to prevent perturbations caused by the surface tension of the water with the film [16]. Fig. 1c shows that the distance between the upper film edge and water surface was $1 \mathrm{~cm}$. Next, the gantry was rotated $15^{\circ}$ with respect to the film to prevent the disturbance of the proton beam path by the film. When the film was in the plane composed of the beam axis, perturbation occurred owing to the EBT3 film's lack of water equivalence for proton beams and the interruption of the beam path by different materials. These factors caused inaccuracies in the range verification owing to dosimetric perturbation. To avoid the aforementioned issue, the gantry can be rotated by a small amount, such as $3^{\circ}$ or $5^{\circ}[7,8,16]$. However, protons penetrate through tissues in a relatively straight manner [17], and therefore, rotating the gantry by the maximum angle would yield more accurate measurements, as most of the beams would travel in water rather than in the EBT3 film. An angle of $15^{\circ}$ was determined to be sufficiently large while ensuring that measurements were not affected at the edge by the penumbra of the $10 \times 10 \mathrm{~cm}$ field.
Two factors could compensate for the perturbations: the gap between the film edge and water surface and oblique beam irradiation. A geometrical correction for the depth distance should be applied to verify the range of each proton beam with the minimum error, as follows:

$$
R_{\text {corr }}=\left(R_{\text {film }}+1\right) / \cos \theta \text {. }
$$

Here, $\theta$ is the correction angle, which is the angle between the beam axis and the film; $R_{\text {film }}$ is the original depth measured with the film; and $R_{\text {corr }}$ is the corrected depth (cm). As the film cannot be positioned $90^{\circ}$ to the ground with a specified accuracy, $\theta$ was subsequently optimized using multiple data with various energies and dose intensities.

The films were scanned twice for background subtraction, once before and once after irradiation, at the same location and position on the scanner bed. The post-irradiation scan was performed 48 hours after irradiation using an Epson 11000XL flatbed scanner (Seiko Epson Co., Tokyo, Japan). Post-irradiation film analyses, including background subtraction and dose conversion with LET correction, were performed using MATLAB version 9.0, R2016a (MathWorks Inc., Natick, MA, USA).

\section{Characteristic curve}

For a read-out process, a characteristic curve must be 


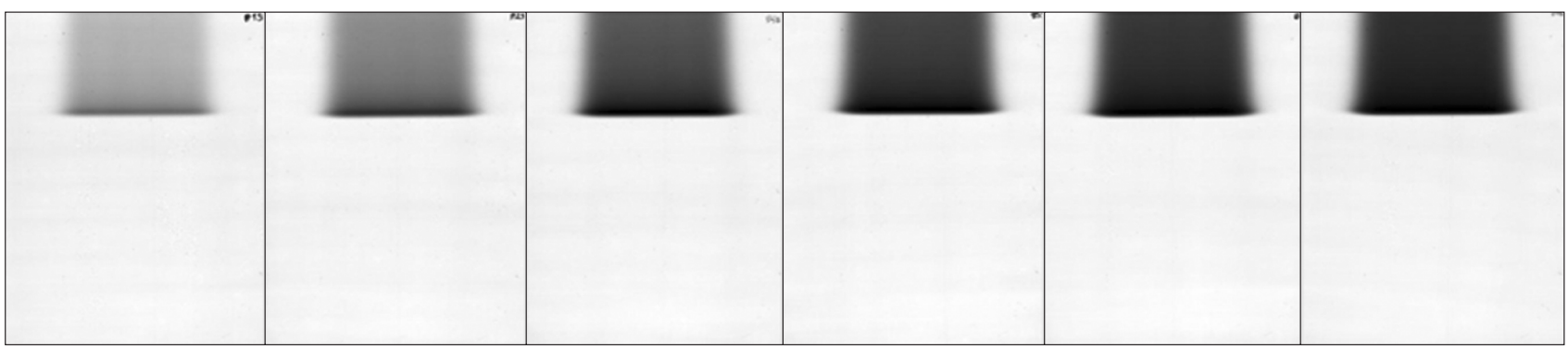

Fig. 2. Scanned film irradiated by 112-MeV beams, showing pristine Bragg peaks with six different dose intensities.

determined. Because the film response depends on the energy and intensity of the proton beam, the formalism used to convert the film response to the absorbed dose is dependent on both variables. First, the 1D relation between film response and absorbed dose for a fixed LET value was determined, and the LET dependence was subsequently considered.

A PPC05 parallel plate-type ionization chamber (IBA dosimetry, Schwarzenbruck, Germany) was used to measure the absolute point dose in the water at a specified depth. Measurements were performed for 112-MeV beams with six different dose intensities at a depth of $2 \mathrm{~cm}$, which is the plateau region of the pristine Bragg peak. The absolute dose could be determined from the direct relationship between film response and absolute dose at a certain depth, namely, at a fixed LET, and Fig. 2 shows the scanned image of the film used to obtain the characteristic curve. By fitting the six plots using Eq. (1), $p, O D_{\max }$, and $D_{1 / 2}$, the characteristic curve at $2 \mathrm{~cm}$ could be determined. However, it is noteworthy that $D_{1 / 2}$ changes with the depth and LET, whereas $p$ and $O D_{\max }$ are constants.

\section{LET calculation and analysis system}

Wilkens and Oelfke [18] have presented a model for the analytical calculation of LET and validated it via Monte Carlo simulations. An analytical calculation of LET was adopted here owing to the fast calculation speed. Although the model of Wilkens and Oelfke [18] does not consider the effect of secondary particles, secondary particles are primarily produced by non-elastic nuclear interactions, which are most significant in the plateau region and negligible in the Bragg peak region $[19,20]$. Wilkens and Oelfke [18] de- scribe the LET as the mean value of the stopping power. In this study, particle fluence appeared at a particular point $x$ and formed an energy spectrum. Therefore, a spectrum of stopping power and its average value were defined as the LET. Eq. (3)-1 represents the track-averaged LET, $L_{t}$, which is the mean value of the stopping power weighted by the particle fluence. In Eq. (3)-1, $r$ is the residual range of each proton at $x$, and $\varphi_{r}(x)$ denotes the spectrum of particles at $x$. According to this model, the LET in water can be calculated analytically for any desired energy with simple parameters, including the standard deviation of the initial energy and the practical range of the beam. Wolfram Mathematica 11 version 11.0.1.0 (Wolfram Mathematica Inc., Chicago, IL, USA) was used to calculate the fluence of the proton beam, stopping power, and track- and dose-averaged LETs.

The SOBP is composed of each of the pristine Bragg peaks along with their respective dose weights. Likewise, the LET of the SOBP was calculated as the weighted sum of each Bragg peak, as expressed in Eq. (3)-2, where $n$ represents the number of beam energies composing one SOBP beam. The LET of the SOBP with three different maximum energies was calculated using this method and plotted in relation to the water depth, as shown in Fig. 3.

$$
\begin{gathered}
L_{t}(x)=\frac{\int_{0}^{\infty} \varphi_{r, j}(x) S(r) d r}{\int_{0}^{\infty} \varphi_{r, j}(x) d r} \\
L_{t}(x)=\frac{\sum_{j=1}^{n} \int_{0}^{\infty} \varphi_{r, j}(x) S(r) d r}{\sum_{j=1}^{n} \int_{0}^{\infty} \varphi_{r, j}(x) d r}
\end{gathered}
$$




\section{LET correction function}

Once the constant values of $O D_{\max }$ and $p$ were obtained from the characteristic curve, $D_{1 / 2}$ could be determined as the LET correction function. For the $150-\mathrm{MeV}$ SOBP beam, the PDD was measured using the MLIC and the film. $D_{1 / 2}$ was directly calculated as a function of depth using Eq. (1), and, on the basis of the corresponding LET dataset, it could be converted to a function of LET. Fifth-degree Gaussian curve fitting was used to derive the formula of $D_{1 / 2}$ (LET), which is applicable regardless of the initial energy of the proton beam provided that the LET of each irradiated beam is identified. Considering the LET dependence of the different energies, the underestimation correction using this method was evalutated in various energy ranges measured as described in Table 1.

\section{Application in the treatment planning system (TPS)}

The correction process was performed for an automated and optimized beam planned by a TPS, unlike the manually planned SOBPs presented in Table 1. One of the treatment plans was exported from the TPS in RayStation version 6 (6.2.0.7; RaySearch Laboratories AB, Stockholm, Sweden) to evaluate the application of the LET correction method to the TPS data. A dose with a uniform cubic distribution was irradiated in water, which is a uniform SOBP plane, with a square beam measuring $10 \times 10 \mathrm{~cm}$ and comprising 34 en-

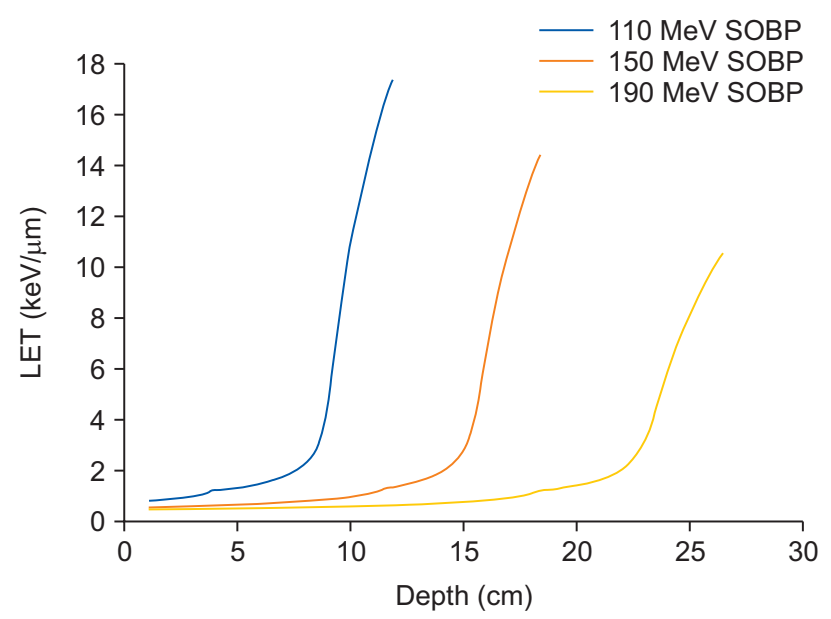

Fig. 3. Analytically calculated linear energy transfer (LET) of spread-out Bragg peaks (SOBPs) with maximum energies of 110, 150, and $190 \mathrm{MeV}$. ergy layers ranging from 81.6 to $155.2 \mathrm{MeV}$. As shown in Fig. 4 , the dose distribution was calculated using the TPS and then compared with the corrected film data. Each layer had a different line spacing and dose distribution, determined automatically from the TPS. The relative dose weights of each energy layer were exported from the treatment plan report to calculate the LET.

\section{Results}

\section{Characteristic curve}

The film responses for the six different dose intensities of the 112-MeV beam and their corresponding doses measured using an ionization chamber were plotted (blue inverted triangle) and fitted (red solid line) with the curve of Eq. (1) (Fig. 5). At a 2-cm depth, the LET was $1.54 \mathrm{keV} /$ $\mu \mathrm{m}$, and the corresponding $D_{1 / 2}$ was $4.21 \times 10^{5}$. It is noteworthy that $D_{1 / 2}$ is a variable. By adjusting the fitting curve,

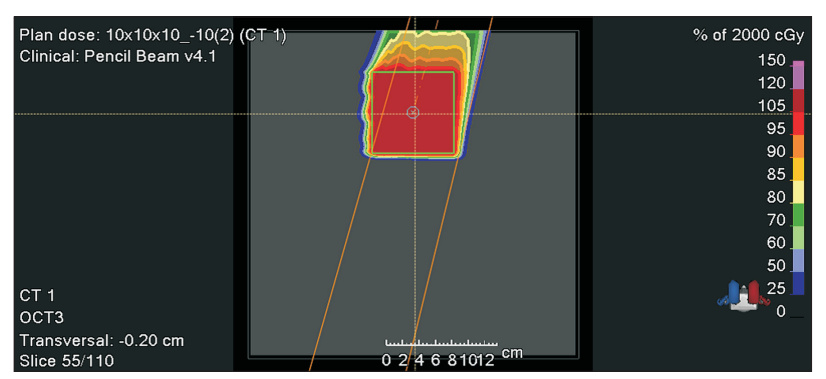

Fig. 4. Dose distribution of cubic beam irradiated obliquely with a gantry angle of $15^{\circ}$ in treatment planning system.

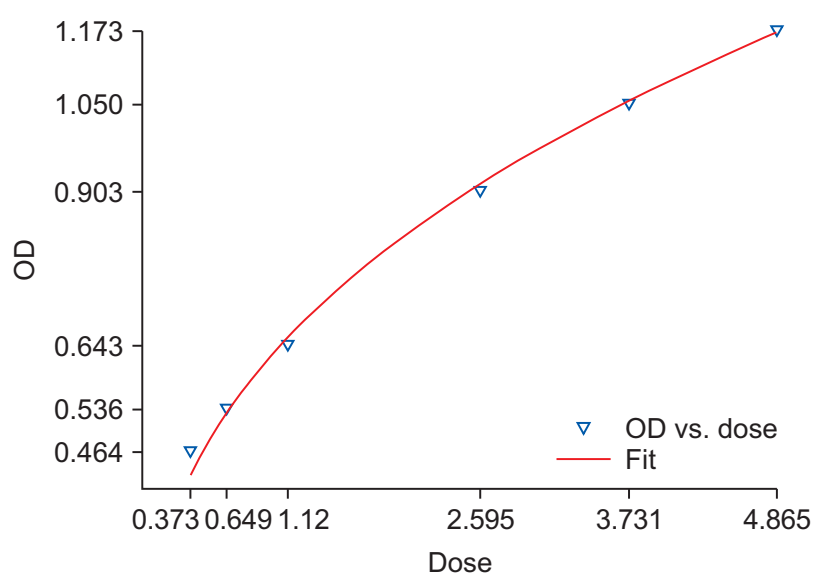

Fig. 5. Characteristic curve of 112-MeV pristine Bragg peak for six different dose intensities at a 2 -cm depth. 
the constant parameters $p$ and $O D_{\max }$ were determined to be 1.049 and 1.586, respectively, with an $R^{2}$ value of 0.9966 , indicating that the bimolecular model is an adequate fitting function to relate the film response to the dose. This further indicates that the bimolecular model is suitable as a characteristic curve.

\section{LET correction function}

$D_{1 / 2}$ was calculated using Eq. (1) and converted to a function of LET. Then, $D_{1 / 2}$ was fitted to a fifth-degree Gaussian curve, as shown in Eq. (4), where $a_{i}, b_{i}$, and $c_{i}$ are curve fitting parameters. The corresponding values are presented in Table 2 and plotted in Fig. 6a. The fitted $D_{1 / 2}$ was then converted to a function of LET for each beam, as shown in Fig. 6b. For all irradiated energies, the LETs along the water depth were calculated. Thus, the $D_{1 / 2}$ for each energy could also be calculated.

$$
D_{1 / 2}(\mathrm{LET})=\sum_{i=1}^{5} \mathrm{a}_{i} \mathrm{e}^{-\frac{\mathrm{LET}-b_{i}}{c_{i}}}
$$

Once $D_{1 / 2}$ was obtained and inserted into Eq. (1), the PDD for each beam could be computed from the $O D$ data. Fig. 7 shows the corrected film measurements (solid line) compared with the PDD measured with Zebra (inverted triangle) for each single-energy beam from 70 to $190 \mathrm{MeV}$. In Fig. 8 , the film measurements of the SOBP are compared before (blue dotted line) and after (green solid line) LET correction

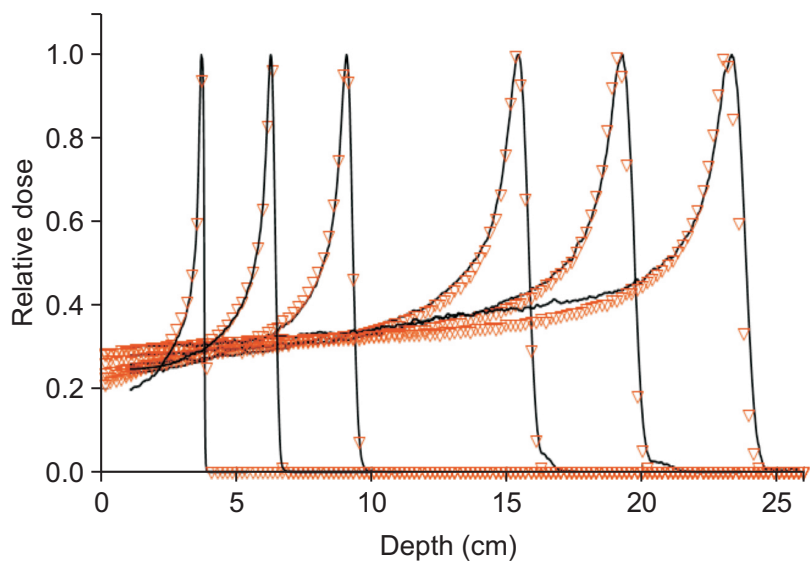

Fig. 7. Pristine Bragg peaks (70, 92, 112, 150, 170, and $190 \mathrm{MeV}$ from left to right) measured using the EBT3 film (corrected; solid line) and those measured using the MLIC (Zebra; inverted triangles).

Table 2. Fifth-degree Gaussian curve fitting parameters, as presented in Eq. (4)

\begin{tabular}{cccccc}
\hline $\mathrm{x}_{\mathrm{i}}$ & $i=1$ & $i=2$ & $i=3$ & $i=4$ & $i=5$ \\
\hline $\mathrm{x}=a$ & $2.95 \times 10^{5}$ & $1.78 \times 10^{5}$ & $3.12 \times 10^{5}$ & $-1.53 \times 10^{5}$ & $4.43 \times 10^{5}$ \\
$\mathrm{x}=b$ & 7.17 & 6.26 & 5.31 & 3.4 & 1.69 \\
$\mathrm{x}=c$ & 0.9 & 1.04 & 1.91 & 0.18 & 5.09 \\
\hline
\end{tabular}
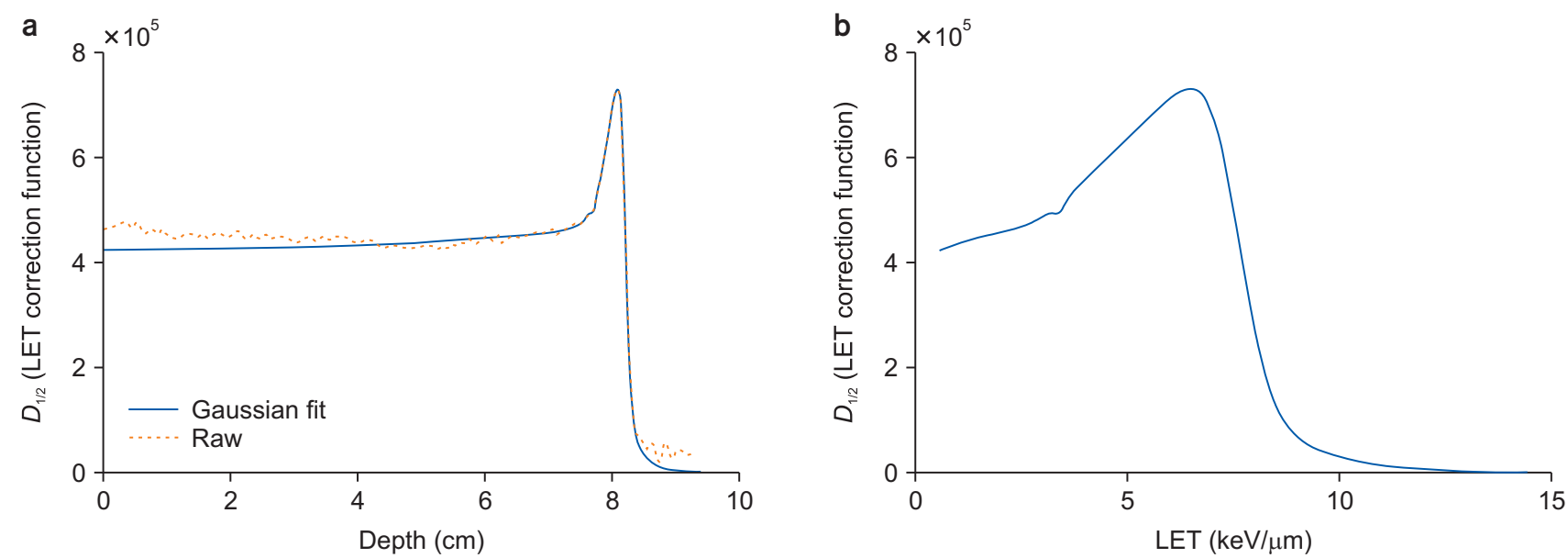

Fig. 6. (a) $D_{1 / 2}$ calculated using spread-out Bragg peak (SOBP) data with a maximum energy of $150 \mathrm{MeV}$ represented along the water depth and the fitted curve based on a fifth-degree Gaussian curve. (b) $D_{1 / 2}$ converted to a function along the linear energy transfer (LET). 
and validated with Zebra (purple ' $x$ ' symbol). The cubic plan of the TPS is shown in Fig. 9. Table 3 shows a comparison of the proton range depth, R80, which is the depth of the proton beam at $80 \%$ of the distal fall-off, for all singleand multi- energy beams to verify the accuracy of the range measured by the EBT3 film. The R80 of the reference used for comparison with that of the EBT3 film was measured by Zebra for pristine Bragg peaks and SOBPs, and the R80 was also calculated using the TPS for the TPS-planned cubic plan. The R80 error was within $0.1 \mathrm{~cm}$. The relative dose discrepancy was used as a criterion to assess the underdose response. When $D_{1 / 2}$ was not considered, the dose underestimation ranged from $14.7 \%$ to $24.68 \%$ for the Bragg peak and $21.7 \%$ to $32.4 \%$ for the SOBP. The bimolecular model correction improved the underdose response by up to $3.89 \%$ of the maximum difference for the single-energy beam and by $3.05 \%$ for the SOBP.

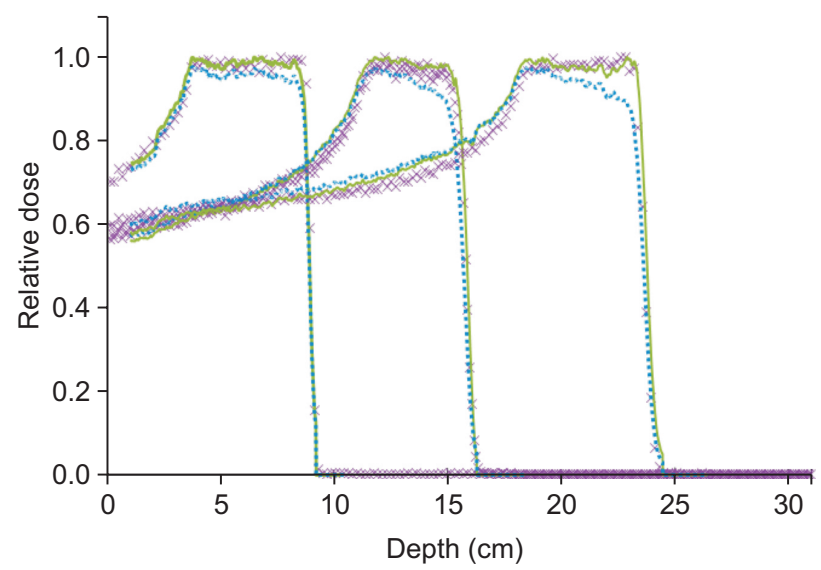

Fig. 8. Depth profile of spread-out Bragg peak (SOBP) measured using the EBT3 film (uncorrected shown as dotted blue line and corrected as solid green line) compared with the multilayer ionization chamber (MLIC) data (purple ' $\mathrm{x}$ ' symbol) for maximum energies of 110, 150, and $190 \mathrm{MeV}$.

\section{Discussion}

The use of the Gafchromic EBT3 film for proton dosimetry has led to significant issues. In particular, the energy or LET dependence of the film has resulted in the underestimation of measured doses at low energies or for high-LET regions. As the bimolecular model is based on chemical reaction laws, which are affected by physical phenomena (known as the LET effect of protons), the performance of previously developed correction methods is adequate when the LET along the depth of the irradiated beam is known.

In this study, the bimolecular model was adapted and successfully extended for application to clinically meaningful multi-energy beams or SOBPs. The LET of a multienergy beam was calculated using an analytical method that considered the dose weight and LET of each single energy beam constituting the SOBP. The model was validated for a clinically important range of energies, from 70 to 190 MeV. The depth-dose distribution of the EBT3 film had an error within $4 \%$, and it demonstrated submillimeter ac-

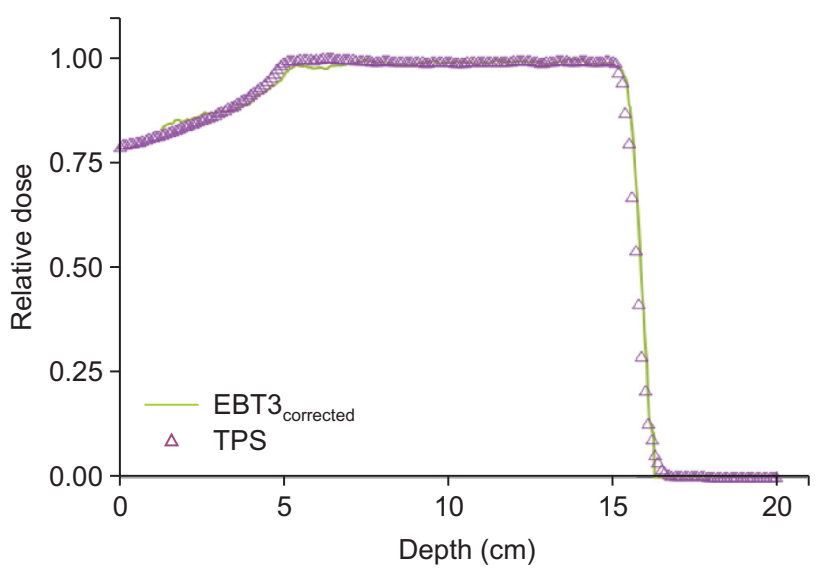

Fig. 9. Depth profile of the cubic treatment planning system (TPS) plan measured with film compared with the TPS data.

Table 3. Range (R80) comparison of single-energy, multi-energy, and TPS-planned beams between the EBT3 film and reference measurements or calculations

\begin{tabular}{clccccc}
\hline Energy & Single-energy & $70 \mathrm{MeV}$ & $92 \mathrm{MeV}$ & $112 \mathrm{MeV}$ & $150 \mathrm{MeV}$ & $170 \mathrm{MeV}$ \\
\hline \multirow{2}{*}{ R80 $(\mathrm{cm})$} & EBT3 & 3.79 & 6.38 & 9.22 & 150.66 & 19.54 \\
& Reference & 3.77 & 6.39 & 9.21 & 15.61 & 19.45 \\
Energy & Multi-energy & SOBP $110 \mathrm{MeV}$ & SOBP $150 \mathrm{MeV}$ & SOBP $190 \mathrm{MeV}$ & & TPS cubic plan \\
R80 $(\mathrm{cm})$ & EBT3 & 8.78 & 15.62 & 23.54 & 15.62 \\
& Reference & 8.85 & 15.54 & 23.45 & 15.53 \\
\hline
\end{tabular}

TPS, treatment planning system; SOBP, spread-out Bragg peak. 
curacy in range verification when compared with MLIC measurements and TPS calculations. The submillimeter accuracy of this method also enables range measurements of unknown beam energies, meaning that, conversely, an unknown beam energy can be calculated by measuring the range. The observed error of range verification contained uncertainties caused by the non-water characteristics of the EBT3 film material, and the effect of secondary particles on LET calculation was disregarded. Hence, the EBT3 film can be used with acceptable uncertainty using the investigated correction method.

\section{Conclusion}

Measuring the depth dose of a dynamically scanned proton beam using an ionization chamber is challenging owing to the extremely time-consuming dosimetry procedure. By constructing a generalized correction method for energy dependence, the EBT3 film becomes an excellent candidate for the depth-dose measurements of scanned beams owing to its efficient measurement procedure, which requires only one beam irradiation, and the high spatial resolution that can be obtained. A high spatial resolution provides excellent usability for low-energy beams that have steep distal fall-offs of the SOBP, such as eye treatment beams. A depth-wise dosimetric quality assurance (QA) is feasible for clinical beams using the EBT3 film. Hence, this could be a promising tool for patient-related QA. However, it still needs to be verified with a non-uniform depth-dose distribution by computing the LET at each location or depth. Calculating the correction parameter for the depth-dose distribution requires the dose weight of each energy along the depth, which can be obtained by analyzing the log file from the TPS. Therefore, further studies regarding nonuniform beams derived from TPS will warrant application to clinical cases.

\section{Acknowledgements}

This research was supported by the National Research Foundation of Korea (NRF) funded by the Ministry of Science, ICT \& Future Planning (2019M2A2B4096537 and 2019R1F1A1062775).

\section{Conflicts of Interest}

The authors have nothing to disclose.

\section{Availability of Data and Materials}

All relevant data are within the paper and its Supporting Information files.

\section{Author Contributions}

Conceptualization: Youngyih Han. Data curation: Moonhee Lee. Funding acquisition: Youngyih Han. Investigation: Moonhee Lee, Wonjoong Cheon, Sunghwan Ahn. Methodology: Moonhee Lee, Wonjoong Cheon. Project administration: Moonhee Lee, Youngyih Han. Resources: Youngyih Han. Supervision: Youngyih Han. Validation: Moonhee Lee. Visualization: Moonhee Lee. Writing-Original draft: Moonhee Lee. Writing-review \& editing: Moonhee Lee, Younghyih Han.

\section{References}

1. International Commission on Radiation Units \& Measurements. ICRU Report 78: Prescribing, recording, and reporting proton-beam therapy. Bethesda: International Commission on Radiation Units \& Measurements; 2009:60-62.

2. Schaffner B, Pedroni E, Lomax A. Dose calculation models for proton treatment planning using a dynamic beam delivery system: an attempt to include density heterogeneity effects in the analytical dose calculation. Phys Med Biol. 1999;44:27-41.

3. Sorriaux J, Kacperek A, Rossomme S, Lee JA, Bertrand D, Vynckier S, et al. Evaluation of Gafchromic ${ }^{\circledR}$ EBT3 films characteristics in therapy photon, electron and proton beams. Phys Med. 2013;29:599-606.

4. Reinhardt S, Hillbrand M, Wilkens JJ, Assmann W. Comparison of Gafchromic EBT2 and EBT3 films for clinical photon and proton beams. Med Phys. 2012;39:5257-5262.

5. Arjomandy B, Tailor R, Anand A, Sahoo N, Gillin M, Prado $\mathrm{K}$, et al. Energy dependence and dose response of Gafchromic EBT2 film over a wide range of photon, electron, and proton beam energies. Med Phys. 2010;37:1942-1947. 
6. Kirby D, Green S, Palmans H, Hugtenburg R, Wojnecki C, Parker D. LET dependence of GafChromic films and an ion chamber in low-energy proton dosimetry. Phys Med Biol. 2010;55:417-433.

7. Zhao L, Das IJ. Gafchromic EBT film dosimetry in proton beams. Phys Med Biol. 2010;55:N291-N301.

8. Perles LA, Mirkovic D, Anand A, Titt U, Mohan R. LET dependence of the response of EBT2 films in proton dosimetry modeled as a bimolecular chemical reaction. Phys Med Biol. 2013;58:8477-8491.

9. Park SA, Kwak JW, Yoon MG, Shin DH, Lee SB, Cho KH, et al. Dose verification of proton beam therapy using the Gafchromic EBT film. Radiat Meas. 2011;46:717-721.

10. Gambarini G, Regazzoni V, Grisotto S, Artuso E, Giove D, Borroni M, et al. Measurements of spatial distributionof absorbed dose in protontherapy with Gafchromic EBT3. Paper presented at: International Symposium on Solid State Dosimetry; 2014 Apr 13-16; Cusco, Peru. p. 742-755.

11. Fiorini F, Kirby D, Borghesi M, Doria D, Jeynes JC, Kakolee $\mathrm{KF}$, et al. Dosimetry and spectral analysis of a radiobiological experiment using laser-driven proton beams. Phys Med Biol. 2011;56:6969-6982.

12. Fiorini F, Kirby D, Thompson J, Green S, Parker DJ, Jones B, et al. Under-response correction for EBT3 films in the pres- ence of proton spread out Bragg peaks. Phys Med. 2014;30: 454-461.

13. Lyman T. Victor schumann. Astrophys J. 1914;38:1-4.

14. Candler C. The photographic process as a diatomic reaction. Aust J Phys. 1960;13:419-436.

15. Candler C. The track density of a charged particle in a photographic emulsion. Il Nuovo Cimento (1955-1965). 1961; 21:1-6.

16. van Battum LJ, Heijmen BJ. Film dosimetry in water in a 23 MV therapeutic photon beam. Radiother Oncol. 1995;34: 152-159.

17. Kanai T, Kawachi K, Matsuzawa H, Inada T. Three-dimensional beam scanning for proton therapy. Nucl Instrum Methods Phys Res. 1983;214:491-496.

18. Wilkens JJ, Oelfke U. Analytical linear energy transfer calculations for proton therapy. Med Phys. 2003;30:806-815.

19. Rosenfeld AB, Wroe AJ, Cornelius IM, Reinhard M, Alexiev D. Analysis of inelastic interactions for therapeutic proton beams using Monte Carlo simulation. IEEE Trans Nucl Sci. 2004;51:3019-3025.

20. Paganetti H. Nuclear interactions in proton therapy: dose and relative biological effect distributions originating from primary and secondary particles. Phys Med Biol. 2002;47: 747-764. 